\title{
Tangence
}

\section{La Bible, un corpus clos d'Écritures}

\section{Jean-Yves Thériault}

Numéro 35, mars 1992

Des écritures à lire

URI : https://id.erudit.org/iderudit/025695ar

DOI : https://doi.org/10.7202/025695ar

Aller au sommaire du numéro

Éditeur(s)

Tangence

ISSN

0226-9554 (imprimé)

1710-0305 (numérique)

Découvrir la revue

Citer cet article

Thériault, J.-Y. (1992). La Bible, un corpus clos d'Écritures. Tangence, (35), 9-23.

https://doi.org/10.7202/025695ar d'utilisation que vous pouvez consulter en ligne.

https://apropos.erudit.org/fr/usagers/politique-dutilisation/ 


\title{
La Bible, un corpus clos d'Écritures
}

\author{
Jean-Yves Thériault
}

Un discours indéfini n'a pas de sens; s'il est sans fin c'est qu'il est sans principe. ${ }^{1}$

L'usage d'un terme au singulier, la Bible, pour désigner une réalité plurielle, trois collections de longueur variable d'Écritures saintes - juives, protestantes, catholiques - , signale la particularité de cette œuvre littéraire publiée à des millions d'exemplaires. La Bible constitue en réalité une bibliothèque de quelques dizaines de livres très diversifiés ${ }^{2}$. En la parcourant, on est vite frappé par la diversité de ton et de contenu. D'un point de vue strictement littéraire, il n'y a pas d'unité biblique, mais plutôt un agrégat de textes souvent mal agencés.

La Bible est pourtant traditionnellement reçue comme un tout, tant par les communautés croyantes que dans l'imaginaire collectif qu'elle a marqué de son empreinte. Elle comporte une clôture explicite, un canon propre à chaque tradition religieuse, qui précise de façon rigoureuse la liste des livres reconnus comme inspirés et normatifs. Je ferai d'abord quelques réflexions sur ce processus de canonisation en tant qu'elaboration d'un corpus clos d'écritures autorisées. Je montrerai ensuite quelques effets de ce phénomène d'inclusion et d'exclusion pour la lecture des Écritures.

\section{La formation du corpus biblique}

\subsection{La Bible n'est pas UN livre}

Pendant plusieurs siècles, les Écritures saintes se sont présentées comme un ensemble de rouleaux entreposés dans des coffrets séparés. Nul ne songeait à désigner globalement ces

1 E. Pousset, "La Bible comme univers", Le canon des Ecritures, Paris, Cerf, coll. - Lectio Divina •, n 140,1990 , p. 514.

2 Voir l'article de Paul-André Giguère sur les genres littéraires bibliques. 
écrits. On devait plutôt procéder par énumération des livres visés. Ainsi, au premier siècle avant J.-C., le préfacier de $L a$ sagesse de Ben Sira emploie trois termes pour désigner les livres saints: "la Torah, les Prophètes et les autres écrits." Les écrivains chrétiens des premiers siècles de notre ère utilisent également des appellations collectives: "les évangiles et les lettres apostoliques." Le mot singulier bible vient du pluriel ta biblia, expression grecque qui désigne les livres de la bibliothèque devenue canonique à partir des troisième et quatrième siècles.

C'est à cause de phénomènes relativement récents et qui lui sont plutôt extérieurs qu'on en est venu à considérer vraiment la collection des écrits sacrés comme un livre. La découverte de l'imprimerie a fait que des textes, qui exigeaient autrefois plusieurs rouleaux ou codices, ont pu être reproduits rapidement en édition complète de quelques tomes seulement. La qualité actuelle du papier et des reliures permet de posséder en un seul volume tous les textes bibliques. Chose impossible aux Anciens, quelqu'un peut maintenant brandir un seul livre et dire: "Je crois à la totalité du canon des Écritures. .

\subsection{De la parole à l'écriture}

Le mot canon vient du terme grec kanôn. Il désigne originellement une * canne ", une tige droite de roseau. On utilise cette * canne. pour tenir droit, pour niveler, pour indiquer la bonne direction sans plier. Par dérivation, le sens est passé de l'objet utilisé comme instrument de mesure à la fonction elle-même de règle, de critère, de norme dans divers domaines. Ce terme ne fut appliqué aux livres saints qu'à partir du quatrième siècle de l'ère chrétienne.

Aujourd'hui, l'expression *canon des Écritures* renvoie à la Bible en tant que collection des livres divinement inspirés et reconnus, en vertu de cette origine divine, comme règle infaillible de la foi et norme de l'agir. Des groupes croyants la lisent comme principe de leur rassemblement, y puisant la force de vivre. Cet usage s'enracine dans une démarche communautaire qui remonte aux prophètes israélites. Quand les oracles de ces porte-parole de Yahvé sont mis par écrit et proclamés lors des assemblées cultuelles, les documents porteurs sont perçus comme source d'une communication divine. Se crée ainsi l'idée que Dieu parle dans un écrit. Une scène du livre d'Ézéchiel $(2,8-3,4)$ évoque cette 
croyance: dans une vision, le prophète mange un livre; si ce geste peut symboliser l'absorption de la Parole de Dieu, c'est qu'on a la conviction que le mystère divin peut se couler dans une écriture.

Une partie de la parole prophétique est devenue livre. De même, la prédication apostolique subsiste dans les évangiles écrits. Par rapport à la mobilité de la parole, les recueils écrits constituent une première fixation de la communication vivante. Le passage de l'oralité à la textualité transforme le mode de communication. La parole proférée agit dans l'inter-relation et elle se modifie selon l'évolution des conditions de l'échange. La mise par écrit fige la parole dans une formulation linguistique: elle ne peut plus évoluer en fonction des locuteurs. Stabilisée dans une écriture, elle est désormais soumise à la sagacité du lecteur.

Ce phénomène s'observe largement dans les origines chrétiennes. Le mouvement amorcé par Jésus donnait de l'importance à l'oralité. Dans l'enthousiasme primitif, l'audace de la parole transmise par les disciples immédiats du Seigneur agissait comme signe de sa présence. Avec la dispersion et la mort des premiers témoins, cette mystification de la parole vivante fit place à la nécessité d'une perpétuation de la mémoire dans l'écrit. Peu à peu, la fluidité orale cédait du terrain à la stabilité textuelle, la représentation littéraire du passé s'installant comme référence autorisée à la place de l'audition de la tradition orale vivante. En quelques générations, la référence essentielle pour établir l'identité des églises primitives se modifia deux fois: de la personne et du message de Jésus au corps des témoins apostoliques, puis à un corpus d'Écritures.

Ce passage de l'oral à l'écrit entraîne un double effet: il enregistre une première sêlection, car une grande quantité de la parole se trouve éliminée dans ce processus; il réalise aussi une première fixation de la tradition vivante. C'est le premier pas du mouvement conduisant à la canonisation des textes bibliques.

\subsection{Formation de recueils}

Nous savons que la communauté juive postexilique pratique la réception et la conservation de documents avec tout le respect dû à la Parole de Dieu. Des textes sont au centre de la vie communautaire: ils servent de points de repère et de normes pour la 
12

foi et la conduite. Au temps d'Esdras (vers 400 avant J.-C.), une grande cérémonie de lecture publique ( $\mathrm{Ne} 8$ ) montre que le concept d'écriture sacrée est devenu une réalité dans la communauté juive. Les *livres de Moïse * servent de code normatif pour réguler la foi et l'existence. Les collections prophéticohistoriques prennent le relais de la verve prophétique qui a perdu de sa vigueur. Lieu de rencontre vivante avec les exigences de l'alliance, des écrits font comprendre les sens des événements contemporains et réalisent une interprétation pratique de la Torah.

Un mouvement de sacralisation de documents réputés contenir la Parole de Dieu engendre leur mise à part, leur conservation et leur transmission. La catégorie des Écritures est créée, dans lesquelles la communauté juive cherche son identité. Leur interprétation produit des relectures ${ }^{3}$ qui s'expriment dans de nouvelles créations littéraires. Ces textes forment l'ossature sur laquelle se charpente de plus en plus la vie du peuple. Ils éclairent ses origines, assurent son homogénéité, donnent règles et signification pour la vie présente.

Dans les communautés chrétiennes, la réception des Écritures juives joue un rôle capital. Un processus semblable à celui qu'on a observé dans la tradition juive donne naissance au Nouveau Testament. Pour les besoins de la prédication et de la catéchèse, de la prière et des célébrations liturgiques, de l'exhortation et de la consolation des croyants, des textes divers sont progressivement composés et circulent sous la responsabilité des disciples de la première heure. Regroupés en évangiles par des écrivains théologiens, des livrets de catéchèse historique sur le Seigneur s'ajoutent aux nombreuses lettres apostoliques pour servir de support et de contrôle à la foi, pour guider les nouveaux convertis au Seigneur Jésus dans le monde cosmopolite gréco-romain. Des recueils deviennent plus autorisés que d'autres pour régler la foi nouvelle et régir les comportements dans les diverses circonstances de la vie. Ils sont considérés à l'égal des Écritures juives et reçoivent la même valeur normative, prenant le relais de la parole vivante pour modeler la foi et la vie communautaires.

3 Voir les articles de Jérôme Longtin et de Jean-Jacques Lavoie. 


\subsection{Sélection des livres et délimitation des recueils}

Le processus de formation de la collection des Écritures juives et chrétiennes s'échelonne sur quelques siècles. Je me limite donc a la présentation des facteurs et critères qui ont joue dans l'élaboration du canon chrétien.

L'Église n'a pas inventé la notion d'Écritures. Celles qu'elle avait reçues d'Israël, elle les a rebaptisées Ancien Testament. Elle a élaboré uñ autre corpus en lui conférant peu à peu le même statut d'Écriture normative et en lui donnant le nom de Nouveau Testament.

La situation de l'Église n'est pas la même par rapport à ces deux corpus. Elle reçoit le premier comme un héritage, au nom de la valeur prophétique qu'il possède par rapport à l'événement du Christ.[...] Le rapport de l'Église au second corpus qui va prendre le nom de Nouveau Testament est tout différent, puisque l'Église le précède: c'est elle qui en compose les textes a l'epoque apostolique. ${ }^{4}$

Ce nouveau canon témoigne d'un événement central, le drame pascal du Seigneur, autour duquel se construit une nouvelle identité communautaire.

\subsubsection{Facteurs}

L'élaboration du canon chrétien est une œuvre de discernement collectif né du besoin d'une autorité équivalente à celle du Seigneur. Trois phénomènes accentuent cette exigence:

1. La recherche d'une norme vérifiable de la parole de Dieu trouve sa réponse dans la reconnaissance des documents de l'époque apostolique comme équivalent de la tradition orale issue du Seigneur. Cela entraîne un mouvement de conservation et de diffusion de ces Écritures autorisées.

2. Puisque des écrits sont reconnus comme comme source et garantie de l'autorité permanente de Jésus-Christ, il convient d'en préciser la liste. Les premières hérésies accélèrent le processus de délimitation. Le mouvement de Jésus a donné naissance à deux types d'évangiles: des ensembles plutôt narratifs comme

4 B. Sesboue, a Essai de théologie systématique sur le canon des Ecritures *, Le canon des Ecritures, op. cit., p. 523-524. 
l'Évangile de Marc et des collections de paroles du Seigneur comme l'Évangile de 'Thomas's. Le genre *dits du Seigneur * triomphait dans la gnose hérétique, il ne fut donc pas retenu dans le canon. Par ailleurs, la valorisation scripturaire des écrits évangéliques mettait en cause l'utilité et la validité des Écritures juives. Il fallut revoir leur statut.

3. La volonté de maintenir vivante l'autorité des grandes figures de la première génération chrétienne entraîne l'expansion de la pratique de la pseudonymie. Le foisonnement de la littérature chrétienne et l'apparition de plus en plus fréquente d'œuvres pseudépigraphes $^{6}$ font sentir la nécessité d'une stabilisation du recueil normatif. On doit faire un tri dans cette abondante littérature d'inégale valeur, en respectant plus ou moins consciemment quelques critères.

\subsubsection{Critères}

Tant pour Israël que pour l'Église, la conscience des origines s'avère un élément fondamental. L'exode, pour l'Ancien Testament, le drame pascal, pour le Nouveau, fondent la formation des recueils et la canonisation des écrits. L'idée de l'impossibilité de reprendre l'origine fondatrice sous-tend ce premier critère. La conformité avec l'époque originelle s'impose aux générations subséquentes pour déterminer l'identité actuelle du groupe porteur de la collection sacrée. La canonisation réalise donc une césure temporelle entre une époque fondatrice reconnue comme normative et les siècles postérieurs.

Un autre critère non moins délicat à manier est celui de l'appréciation du contenu d'un écrit en fonction de sa conformité avec la grande tradition apostolique déjà reconnue dans la

5 Cet évangile apocryphe constitue une collection de dits de Jésus. Il existe intégralement en version copte, dans un codex appartenant à la bibliothèque gnostique découverte près de Nag Hamâdi en Égypte. Il s'agit d'un recueil de 114 paroles de Jésus présentées parfois sous la forme de brefs dialogues. Il témoigne de l'existence de cette forme d'évangile.

6 La pseudépigraphie consiste dans le fait qu'un auteur anonyme ou peu connu attribue son écrit à un personnage dont l'autorité est reconnue dans la communauté. Dans la tradition ancienne cela pouvait représenter un effort authentique de perpétuer la pensée de cet ancien et ne constituait pas nécessairement un subterfuge ou une tromperie. 
majorité des églises. Ce critère présuppose donc un certain consensus sur les composantes essentielles de l'héritage reçu. Si le contenu d'un ouvrage comporte des déviations, le patronage apostolique s'avère fallacieux et le livre doit être rejeté du groupe canonique.

La conservation et l'usage constant des textes par les premières générations de croyants constituent le critère fondamental. À travers les cataclysmes successifs, des manuscrits ont survécu; le travail des rédacteurs a protégé des morceaux de traditions; le labeur des scribes a produit des copies et établi des éditions autorisées; des prédicateurs et des missionnaires ont répandu des textes; tout ce processus de sélection progressive fut perçu comme providentiel. Dans la mesure où un texte avait survécu, qu'il avait été révisé, corrigé, édité avec soin pour les besoins de la communauté, on lui accordait une fonction et un statut particuliers: on voyait en cela le signe qu'il était œuvre divine, donc digne de devenir normatif et régulateur dans les églises issues de cette tradition.

\subsection{En guise de corollaire}

Le choix des œuvres canoniques compose certes une œuvre littéraire, mais il énonce surtout les traits essentiels de la rencontre historique de Dieu avec un peuple croyant. L'histoire du canon correspond au processus d'interprétation par l'Église de sa propre identité; elle dessine les caractéristiques de la communauté qui reçoit ces écrits et qui s'en abreuve; elle représente les symptômes de l'Église comme la création porte les traces du Créateur.

La notion de canon engendre le qualificatif biblique: elle rend possible des termes comme "auteurs bibliques ", "monde biblique ", "exégèse biblique ", etc. Les écrivains sacrés ne sont donc pas des auteurs bibliques au sens où ils auraient composé un morceau de l'ensemble biblique comme le font aujourd'hui les collaborateurs d'un ouvrage collectif. Au contraire, chaque livre avec son histoire de composition et de transmission a établi sa propre crédibilité dans la communauté lectrice: par l'usage qu'elle en a fait, celle-ci a instauré la valeur des livres qui composent la Bible.

La canonicité n'indique pas d'abord la qualité littéraire d'un écrit mais la fonction jouée dans la vie communautaire. Reconnue 
16

après coup, elle n'est cependant pas fantaisiste, elle signale et proclame une crédibilité acquise qu'elle perpétue. Si des amalgames de lois et de récits populaires, des collections de paroles prophétiques et sapientielles, des instructions de prédicateurs et des lettres apostoliques, si tout cela a été conservé et transmis, c'est que ces écrits valaient au-delà de la communauté immédiate pour laquelle ils avaient d'abord été produits. Considérés comme textes fondateurs, leur conservation est due à la subsistance des groupes qu'ils rassemblent et font eux-mêmes durer.

\section{Signification du canon}

\subsection{La fonction des Écritures}

La qualification canonique donnée à un écrit signale une double réalité. Elle indique sa réception officielle dans une communauté, constituant une collection close de livres dont on peut faire une liste exhaustive. D'un autre point de vue, le terme canonique certifie la valeur propre au livre ainsi désigné: il est reconnu comme inspiré, apte à réguler la pensée et les comportements du groupe croyant, à agir comme fondement structurant de la collectivité et comme source de nouvelles explications.

Toutes les églises professent la nécessité de la délimitation d'un corpus autorisé. Cela ne donne cependant pas lieu à un canon unique, ni à une pratique uniforme dans la réception des collections. La diversité se situe sur le plan de l'étendue de l'espace textuel reconnu, mais cette différence influence la composition et le caractère de la communauté croyante qui l'assume pleinement, car le canon n'est pas seulement une anthologie de littérature vénérable. Sa clôture délimite le champ où se dessine le visage général du peuple croyant.

En tant que canon, la Bible s'impose comme lieu fondamental de référence et point de repère pour la vérité de l'Église. Celle-ci ne peut élargir le canon selon ses propres volontés; elle ne fait que le recevoir car *ce n'est pas elle qui le constitue: elle a été constituée par lui*7. On ne peut reprendre l'origine fondatrice, ni la modifier, ni la corriger, au risque de se dénaturer. La Bible fournit ainsi le cadre dans lequel tout événement affectant 
la collectivité chrétienne doit être lu et compris. Face aux problèmes existentiels posés par la vie dans un monde en évolution, la communauté croyante se tourne vers ces écrits témoignant de ses origines comme vers un miroir où se reflètent ses caractéristiques fondamentales et où se lisent des orientations essentielles pour sa survie.

Considérer la Bible du point de vue du canon, c'est enregistrer ce fait que des communautés croyantes ont la conviction d'écouter la Paróle divine dans une collection de livres qui fonde leur existence collective et oriente de façon décisive leurs actions. En fait, c'est prendre au sérieux le texte biblique lui-même, car il désigne souvent un énonciateur divin comme source de ses énoncés. C'est lire en respectant le type de validité et d'autorité que ce texte réclame en interpellant son lecteur et en se présentant comme porte-parole de Dieu. Le lecteur croyant accorde véritablement à l'écriture biblique le statut que celle-ci revendique dans son énonciation.

La Bible acquiert son autorité dans un processus de communication, dans la mesure où elle est lue avec la conviction qu'elle peut communiquer du vrai sur l'expérience humaine dans sa rencontre avec un monde qui la dépasse. Accepter la Bible comme Écritures c'est se référer à ce vaste corpus reconnu comme un univers de parole fondamentalement cohérent et significatif. Cela constitue une décision prise sur le sens du monde et de l'histoire, une décision qui privilégie les Écritures comme espace de recherche d'une explication sensée de notre univers.

\subsection{La clôture du canon}

La décision de clore le canon constitue un acte théologique important comportant un double effet: 1. il impose des bornes, un périmètre à la collection des écrits autorisés; 2 . il indique un centre, une certaine structuration de la collection autour d'une unité à découvrir. Tracer le périmètre d'une totalité structurée, c'est produire inclusion, exclusion, mais aussi ouverture.

\subsubsection{Inclusion}

Le canon constitue une enceinte qui cherche à rassembler tout le témoignage utile, à ne rien laisser perdre des textes qui 
attestent de la Parole de Dieu. Ce rassemblement sélectif réalise une convergence d'œuvres indépendantes et même assez disparates. Leur réunion dans cet enclos fait que ces œuvres ne peuvent plus être lues de façon indépendante. Le canon constitue un corps d'écritures, cette image suggérant que le regroupement des livres à l'intérieur du même recueil crée entre eux une solidarité signifiante et permet des lectures structurées et cohérentes.

La Bible chrétienne propose une gestion structurée de la temporalité. Elle a un commencement unique (Genèse) et une fin spécifique (Apocalypse). De plus, la réunion des deux Testaments en une totalité canonique empêche de les lire sans tenir compte de leurs rapports réciproques. Elle engendre une démarche herméneutique originale, basée sur des principes de lecture spécifiquement inspirés du seuil décisif entre deux blocs testamentaires: typologie, promesse-accomplissement, voilé-dévoilé, etc. L'aller et le retour dans le temps sont cependant orientés vers la réalisation en Christ. L'Ancien Testament se révèle comme source et prophétie anticipant la personne et les événements de la vie de Jésus reconnu comme le Messie attendu. Le Nouveau Testament est lu comme interprétation et accomplissement des Écritures vétéro-testamentaires. L'ajout du Nouveau Testament ne fait pas seulement allonger le corpus des Écritures, il transforme la conception des textes sacrés: ils sont désormais reçus comme Écritures accomplies.

\subsubsection{Exclusion}

Le canon installe aussi une frontière. D'une part, il constitue les Écritures canoniques déterminant les seuls livres susceptibles de fonder les enseignements théologiques. Mais il place, d'autre part, des écrits à l'extérieur du périmètre, créant la notion d'œuvres apocryphes. Cette exclusion de la collection autorisée marginalise des écrits parents ou voisins. Appelés apocryphes ou pseudépigraphes, ces ouvrages sont perçus comme sans garantie, suspects ou franchement hérétiques. La délimitation canonique comporte alors un effet de condamnation d'une littérature évaluée comme menaçante et dangereuse ${ }^{8}$. Ces livres a à ne pas lire " seraient contaminés par des courants hérétiques, mensongers par

8 E. Junod, - Choix des écritures chrétiennes et clôture du canon *, LumVie, 171 (1985), p. 15. 
leur titre et leur contenu. Il est possible que des textes transmettant de précieuses traditions et que des témoignages authentiques aient été écartés du canon. Un clivage trop manichéen engendre une opposition entre des documents dont les rapports historiques sont plus complexes. La formation du canon et sa clôture n'ont d'ailleurs pas empêché complètement la lecture, la conservation et la diffusion de ces livres apocryphes que certaines publications récentes font revivre?

\subsubsection{Ouverture}

Le corpus biblique lui-même érige en figures certains événements et personnages de l'histoire vécue du peuple élu. Il se présente ainsi comme un champ ouvert à la lecture où le contenu est à interpréter dans un discours toujours renouvelé. L'exégèse des textes bibliques a d'ailleurs engendré des ensembles complexes de lecture comme le corpus patristique ${ }^{10}$ et le corpus thomiste, ce dernier étant devenu une sorte de canon théologique dans la tradition catholique.

Comme le voyageur suit des itinéraires qui répondent à ses goûts ou à ses intérêts, le lecteur biblique peut être guidé dans certains parcours privilégiés. Le plus évident est celui que présente le calendrier liturgique avec son lectionnaire: un choix de textes en fonction des fêtes et du déroulement de l'année liturgique. On pourrait également retracer des parcours thématiques: ainsi le schéma de l'histoire du salut amène à sélectionner les textes en fonction des suites promesse-réalisation ou attente-accomplissement. Le travail théologique opère une autre forme de sélection et d'organisation en fonction de la valeur explicative ou démonstrative des textes soutenant telle doctrine. On peut même réaliser des parcours esthétiques, les images et les scènes étant choisies en fonction de leur force évocatrice et suggestive. L'iconographie a largement privilégié les évangiles de l'enfance qui ne représentent pourtant qu'une mince section de deux des quatre évangiles.

La Bible sans lecteur reste lettre morte. Elle prend sens et valeur dans les groupes historiques qui la reçoivent, la lisent et l'interprètent. La parole transmise par le texte biblique se

9 Par exemple, les livres de G. Messadié sur Jésus et Paul.

10 Voir l'article de Rodrigue Bélanger. 
20

construit dans le mouvement d'interprétation des traditions anciennes en fonction de nouvelles situations culturelles. En articulant le texte biblique avec des problématiques contemporaines, l'entreprise herméneutique opère des sélections et elle produit du sens nouveau. Elle garde vivant le texte biblique en l'ouvrant à des significations inédites. L'identité de la Bible réside certes dans le contenu qu'elle véhicule, mais elle relève tout autant de la manière dont elle est lue, car le sens se construit dans l'histoire des lectures et des interprétations. Les nouvelles approches issues du domaine littéraire favorisent une -démocratisation - de l'accès au sens et elles ouvrent sur des interprétations moins cléricales comme, entre autres, les lectures féministes.

\subsection{Le centre du canon}

Les Écritures ne se présentent pas comme un document théologique unifié. Elles ne formulent pas une information systématisée des données de la révélation. Elles témoignent des multiples modalités de l'alliance de Dieu avec son peuple et du salut définitif en Jésus-Christ. Michel de Certeau rappelle d'ailleurs que la * surface articulée mais non unifiée (du canon biblique) obéit [...] à un autre type de cohérence que le discours philosophique. Elle ne ramène pas le multiple à l'un. Au contraire, le pluriel est maintenu *11. La normativité du canon s'exprime dans la pluralité des témoignages et dans la diversité des cultures. Cette variété des expressions de la Parole divine dans l'éventail des livres canoniques est un signe de la richesse multiforme selon laquelle cette Parole peut être reçue avec authenticité dans la pluralité des incarnations historiques.

La Bible chrétienne est hétérogène, elle n'est cependant pas hétéroclite. La canonicité affirme qu'on ne doit pas la réduire à un agrégat informe de livres réunis par hasard en une série indéfinie: La lecture des écrits du corpus biblique doit suivre des parcours qui présentent une certaine cohérence. Cette exigence commande la recherche de principes herméneutiques qui permettent de discriminer le normatif du culturel, ce qui doit être structurant pour toutes les générations dans toutes les cultures et ce qui reste lié à des pratiques sociales ou ecclésiales relevant d'un moment particulier. Sans tomber dans les harmonisations 
superficielles et sans prétendre à l'interprétation définitive, il importe de chercher un principe intégrateur ou de retracer un fil conducteur capable de faire tenir les morceaux dans une lecture cohérente. Deux grandes lignes de solution apparaissent.

D'abord reconnaître un développement linéaire à travers l'ensemble biblique: montrer des étapes et des rebondissements dans l'élaboration d'une doctrine, manifester l'évolution de certaines convictions morales avec des tâtonnements et des impasses, percevoir des idées fondamentales qui se développent selon des facettes diversifiées liées aux cultures et aux contingences historiques. Mais surtout, on doit savoir que la cohérence biblique n'est pas donnée, elle est à construire à partir des diversités et des oppositions inhérentes à toute parole humaine. La manifestation d'un dessein divin est à saisir dans un effort de structuration des éléments reconnus comme essentiels par un large consensus ecclésial.

La lecture biblique opère nécessairement des nouveautés. La normativité canonique n'empêche pas le changement. Elle le contrôle en le maintenant dans des limites acceptables. La règle empêche la fantaisie et assure le maintien d'une identité. C'est dans une pratique ecclésiale, et non dans un contrôle purement autoritaire, qu'elle doit jouer si elle veut garder sa fécondité. La diversité dans le canon rappelle la nécessité de critiquer continuellement les interprétations de la Bible en étant attentif aux grands courants de la tradition vivante. La signification des Écritures n'est pas donnée, elle est à construire dans un travail de lecture jamais achevée.

\subsection{Leçons à tirer du canon scripturaire}

Reconnaître une collection d'écrits comme porteuse de la parole divine, c'est l'accepter comme médiatrice dans la communication de cette parole. En ce sens, le texte signale un manque qu'il se propose de combler, à la manière d'une lettre qui supplée à l'absence d'un ami par la présence d'un texte à lire. La lettre tient lieu de la personne aimée et réalise un certain mode de présence, mais elle n'élude pas complètement le sentiment du manque. On peut évoquer J. Derrida et parler de l'écriture comme trace entre l'absence et la présence. Ainsi le texte canonique rend possible l'écoute de la parole de Dieu dans l'Écriture, il offre une forme de présence de ce locuteur, il en atteste le 
vouloir durer dans l'espace. Mais il signale aussi le manque de cette présence directe que d'aucuns pensent trouver dans la Bible. De lui-même le texte ne parle pas: il est en attente d'un sujet lecteur qui le reçoit et l'écoute comme une parole. Dans la Bible, la Parole de Dieu est en attente du sujet en qui elle va parler et prendre corps.

Il y a risque de mal lire la Bible, si on lui accorde une valeur normative au point de la considérer comme un donné à reproduire. Elle se présente souvent elle-même comme une relecture de ses propres textes. Le caractère canonique ne dispense pas du travail de lecture dans des conditions historiques changeantes, avec les ressources plurielles des méthodes de lecture en constante évolution. Dans ce va-et-vient continuel du texte à la situation du lecteur se construit la rencontre avec la parole vivante que le croyant espère trouver dans les Écritures.

Professer un canon, c'est choisir un univers de parole et affirmer qu'un discours doit avoir une cloture: "Un discours indéfini n'a pas de sens; s'il est sans fin c'est qu'il est sans principe. ${ }^{12}$ La réciproque est aussi vraie: participer à la vie d'une communauté c'est adopter un canon. "Il est des "écritures" qu'il faut avoir lues pour appartenir à un groupe. ${ }^{13}$ Cela se vérifie dans le domaine scientifique où il faut avoir fréquenté des corpus de livres reconnus pour appartenir véritablement à la discipline établie. N'y a-t-il pas des livres qu'il est impardonnable d'oublier lorsqu'on fait des demandes de subvention?

Le refus de tout canon peut sembler un acte de liberté et d'autonomie, mais il entraîne inévitablement une perte de sens. Je ne peux me comprendre si je lis sans repère et sans fin des textes à côté d'autres textes. Choisir ne constitue pas nécessairement un réflexe sectaire. Si les membres d'une société ne peuvent tolérer aucun acte d'autorité venu de l'extérieur, si la seule mesure c'est le goût ou l'opinion individuelle, cette société va vers la dissolution. Qu'arriverait-il à une culture qui ne choisirait rien, où tout serait amassé sans appréciation et sans classement? Tout serait banalisé. Si chaque fragment d'écriture mérite également conservation, aucune ouvre n'a véritablement de la valeur. Une société qui serait régie par le principe d'absence de clôture ingurgiterait

12 E. Pousset, Le canon des Ecritures, op. cit., p. 514.

13 F. Marty, Le canon des Écritures, op. cit., p. 496. 
indistinctement et indéfiniment tous les produits culturels au point de devenir insignifiante. Sans canon, pas d'identité possible, ni d'orientation vers un avenir.

Une communauté humaine qui tient à une identité doit s'engager dans un rapport vivant avec un corpus de traditions qui soit suffisamment large et riche pour assurer un développement créateur. Tenir ensemble suppose une mémoire, corps d'écriture, dont les choix et la clôture ne sont pas à la fantaisie des individus du groupe. ${ }^{14}$ Prendre au sérieux un canon d'écritures, c'est se donner un lieu commun de ressourcement et d'identité collective et, surtout, susciter ce type de lecteurs attentifs à rencontrer ces textes dans toute leur épaisseur afin d'y fonder une production nouvelle de textes signifiants. C'est ce qui fait dire à $F$. Marty: - Notre chance, comme croyants, est d'avoir un rapport vivant avec des textes fondateurs, et nous sommes responsables de la fécondité de ce rapport. . 15

Ces réflexions sur le canon biblique font reconnaître l'importance de textes fondateurs dans les sociétés contemporaines. Dans les grands débats éthiques mondiaux où les enjeux de justice apparaissent comme une condition de survie de l'humanité, les énoncés sur les droits de l'être humain ne représententils pas un - canon des droits fondamentaux et universels *? Dans une perspective plus nationaliste, notre chance comme Québécois ne serait-ce pas d'avoir un rapport vivant à un corpus de traditions avec lequel nous entretenons un rapport fécond et créateur? Pour survivre, la collectivité québécoise doit se délimiter un champ de signification par la référence à un corpus de traditions sociales, littéraires et culturelles identifiable et reconnu, susceptible d'engendrer de nouvelles créations parce qu'il serait générateur de significations.

14 F. Marty, ibid., p. 509.

15 Ibid. 